\title{
THE HOMOLOGY SEQUENCE OF THE DOUBLE COVERING; BETTI NUMBERS AND DUALITY
}

\author{
RUSSELL G. BRASHER
}

0 . The existence of an orientable double covering of a nonorientable manifold has long been known. One of the earlier treatments, for triangulable manifolds, is to be found in [3]. As usually realized, given a nonorientable manifold $X$, the orientable double-covering manifold $\tilde{X}$ corresponds to the "orientation-preserving" subgroup of index two of the fundamental group $\pi\left(X, x_{0}\right)$, this subgroup being defined by the action of the fundamental group on the local homology group $H_{n}\left(X, X-x_{0}\right)$. A more constructive-essentially sheaftheoretic-approach is available which obtains $\tilde{X}$ directly in terms of the groups $\left\{H_{n}(X, X-x) \mid x \in X\right\}$ and which more readily yields certain information about the spaces.

It is the purpose of this note to point out an exact homology sequence associated with the double covering, and to show that it yields a relation between the Betti numbers of the respective spaces and also gives rise to a "duality sequence."

Terminology and notation are standard. An $n$-dimensional manifold, or $n$-manifold, is a second-countable Hausdorff space in which each point has a coordinate neighborhood (nbd) homeomorphic to Euclidean $n$-space $R^{n}$. In this paper all manifolds shall be compact connected manifolds without boundary, and no assumptions of triangulability are made. For standard results in the algebraic treatment of manifolds one may consult Eilenberg-Steenrod [2] or Spanier [4]. Homology and cohomology will refer throughout to the singular groups with integral coefficients or (see $\$ 2$ below) with coefficients in certain local systems.

1. Let $X$ be an arbitrary compact connected nonorientable $n$-manifold without boundary. Then $\tilde{X}$ is the component of the orientation sheaf containing the generators of the stalks. We briefly indicate some of the details.

By excision, $H_{n}(X, X-U) \approx Z$ for any coordinate nbd $U$ in $X$. For any $x \in U$, the homomorphism $\alpha_{u}^{x}: H_{n}(X, X-U) \rightarrow H_{n}(X, X-x)$ induced by inclusion is bijective since $X-U$ is a deformation retract of $X-x$. For any $x \in U$ and generator $e_{u} \in H_{n}(X, X-U), \alpha_{u}^{x}\left(e_{u}\right)$ generates $H_{n}(X, X-x)$. Thus for $x$ and $y$ both in the same coordinate nbd $U$ we may specify an isomorphism $\alpha_{x}^{y}=\alpha_{u}^{y} \alpha_{x}^{u}$ where $\alpha_{x}^{u}=\left(\alpha_{u}^{x}\right)^{-1}$. If $U_{x}$ and $U_{y}$ are coordinate nbds of any points $x$ and $y$, there are finite

Received by the editors May 15, 1969. 
chains $U_{1}, \cdots, U_{k}, U_{1}=U_{x}, U_{k}=U_{y}, U_{i} \cap U_{i+1} \neq \square$, and isomorphisms $\alpha_{x}^{y}=\alpha_{u_{k}}^{y} \alpha_{x_{k-1}}^{u_{k}} \cdots \alpha_{u_{1}}^{x_{1}} \alpha_{x}^{u_{1}}$, where $x_{i} \in U_{i} \cap U_{i+1}$.

Let $X^{*}=\mathrm{U}\left\{H_{n}(X, X-x) \mid x \in X\right\}$; a typical point in $X^{*}$ is $\left(x, z_{x}\right)$ with $x \in X$ and $z_{x} \in H_{n}(X, X-x)$. If $U$ is a coordinate nbd in $X$, let $\left(U, z_{u}\right)=\left\{\left(x, z_{x}\right) \in X^{*} \mid x \in U, z_{x}=\alpha_{u}^{x}\left(z_{u}\right)\right\}$. The collection $\left\{\left(U, z_{u}\right) \mid U\right.$ is a coordinate nbd in $X\}$ is a base for a topology on $X^{*}$, and the function $\rho: X^{*} \rightarrow X$ defined by $\rho\left(x, z_{x}\right)=x$ is a covering map.

Thus $X^{*}$ is locally the product of a nbd $U$ in $X$ and a discrete set in one-to-one correspondence with $Z$, and we may intuitively identify $U$ with $U \times\{0\}$. The space $X^{*}$ is too big and consists of countably many redundant sheets over $X$; questions of orientation are determined by the behavior of the generators of the stalks $H_{n}(X, X-x)$. This leads to the following definition.

Let $x_{0}$ be any point of $X$ and $e_{x_{0}}$ one of the generators of $H_{n}\left(X, X-x_{0}\right)$. We define $\tilde{X}$ to be the component of $X^{*}$ containing the point $\left(x_{0}, e_{x_{0}}\right)$. The restriction of $\rho$ to $\tilde{X}$ will be denoted by $p$. The relevant facts are collected in the following theorem.

(1) Theorem. $\tilde{X}$ is a compact connected orientable n-manifold. $p: \tilde{X} \rightarrow X$ is a covering map of multiplicity two.

2. In this section and the next we consider the homology and cohomology of $X$ with coefficients in local systems arising from the construction of $\tilde{X}$. We first briefly recall the standard definitions.

A local system $\Gamma$ of abelian groups on $X$ is a covariant functor from the fundamental groupoid of $X$ to the category of abelian groups. Thus $\Gamma$ assigns to each $x \in X$ an abelian group $\Gamma(x)$ and to each path $\omega$ from $x$ to $y$ an isomorphism $\Gamma_{\omega}: \Gamma(x) \rightarrow \Gamma(y)$ depending only on the homotopy class of $\omega$. If $\Gamma$ is a local system of abelian groups on $X$, then $\operatorname{Hom}(\Gamma, Z)$ is a local system which assigns to each $x \in X$ the group $\operatorname{Hom}(\Gamma(x), Z)$, and $\Delta^{*}(X ; \operatorname{Hom}(\Gamma, Z)) \approx \operatorname{Hom}\left(\Delta_{*}(X ; \Gamma), Z\right)$. Homology and cohomology groups of $X$ with coefficients in a local system are defined as in [4].

Now the system $\left\{H_{n}(X, X-x), \alpha_{x}^{y}\right\}$ is a local system of abelian groups on $X$, that we will denote by $\Gamma$. Thus $\Gamma(x)=H_{n}(X, X-x)$ and if $\omega$ is a path from $x$ to $y, \Gamma_{\omega}: \Gamma(x) \rightarrow \Gamma(y)$ is the isomorphism $\alpha_{x}^{y}: H_{n}(X, X-x) \rightarrow H_{n}(X, X-y)$ defined previously.

If $\sigma: \Delta^{q} \rightarrow X$ is a singular $q$-simplex of $X, \sigma$ has exactly two liftings $\tilde{\sigma}_{1}, \tilde{\sigma}_{2}: \Delta^{q} \rightarrow \tilde{X}$. The mapping $\mu: \Delta_{q}(X) \rightarrow \Delta_{q}(\tilde{X})$ defined by $\mu(\sigma)=\tilde{\sigma}_{1}+\tilde{\sigma}_{2}$ is a chain transformation $\Delta(X) \rightarrow \Delta(\tilde{X})$. We may also define $\nu: \Delta_{q}(\tilde{X})$ $\rightarrow \Delta_{q}(X ; \Gamma)$ by $\nu(\tilde{\sigma})=e_{x_{0}} p \tilde{\sigma}$ where $\tilde{\sigma}\left(v_{0}\right)=\left(x_{0}, e_{x_{0}}\right) ; \nu$ is a chain transformation $\Delta(X) \rightarrow \Delta(X ; \Gamma)$. 
It is straightforward to verify that $\mu$ is injective, $\nu$ is surjective, and $\operatorname{Im} \mu=\operatorname{Ker} \nu$. Thus we have the short exact sequence of chain complexes

$$
0 \rightarrow \Delta(X) \stackrel{\mu}{\rightarrow} \Delta(\tilde{X}) \stackrel{\nu}{\rightarrow} \Delta(X ; \Gamma) \rightarrow 0
$$

and the corresponding exact sequence of homology groups

$$
\cdots \rightarrow H_{q}(X) \stackrel{\mu_{*}}{\rightarrow} H_{q}(\tilde{X}) \stackrel{\nu_{*}}{\rightarrow} H_{q}(X ; \Gamma) \stackrel{\partial_{*}}{\rightarrow} H_{q-1}(X) \rightarrow \cdots
$$

The sequence (3) displays a nice relationship among the homology of $X$, of $\tilde{X}$, and of $X$ with coefficients in the local system used to construct $\tilde{X}$. We will give two applications of this sequence, the first of which is the following result relating the Betti numbers of $X$ and $\tilde{X}$. Let $\rho_{q}$ be the rank of $H_{q}(X)$ (or of $H^{q}(X)$ ) and $\tilde{\rho}_{q}$ the rank of $H_{q}(\tilde{X})$.

(4) Theorem. $\tilde{\rho}_{q}=\rho_{q}+\rho_{n-q}$.

We first note that, for each $q$, every element of Ker $\mu_{*}$ is of order two. Since $\operatorname{Ker} \mu_{*}=\operatorname{Im} \partial_{*}$, we have $2 H_{q}(X ; \Gamma) \subset \operatorname{Ker} \partial_{*}=\operatorname{Im} \nu_{*}$. This implies $\operatorname{rank}\left(\operatorname{Im} \nu_{*}\right)=\operatorname{rank} H_{q}(X ; \Gamma)$.

Let $\mu_{*}^{\prime}$ and $\nu_{*}^{\prime}$ be the restrictions of $\mu_{*}$ and $\nu_{*}$ to $F_{q}(X)$ and $F_{q}(\tilde{X})$, the free parts of $H_{q}(X)$ and $H_{q}(\tilde{X})$ respectively. Let $N$ be the quotient of $\operatorname{Im} \nu_{*}^{\prime}$ by its torsion subgroup and $\nu_{*}^{\prime \prime}=\eta \nu_{*}^{\prime}$ where $\eta: \operatorname{Im} \nu_{*}^{\prime} \rightarrow N$ is the natural surjection. The sequence

$$
0 \rightarrow F_{q}(X) \stackrel{\mu_{*}^{\prime}}{\rightarrow} F_{q}(\tilde{X}) \stackrel{\nu_{*}^{\prime}}{\rightarrow} N \rightarrow 0
$$

is exact at the ends but not in the middle. However Ker $\nu_{*}^{\prime \prime} / \operatorname{Im} \mu_{*}^{\prime}$ is a torsion group. Thus $\operatorname{rank}\left(\operatorname{Ker} \nu_{*}^{\prime \prime}\right)=\operatorname{rank}\left(\operatorname{Im} \mu_{*}^{\prime}\right)$.

Since $N$ is a free group,

$$
0 \rightarrow \operatorname{Ker} \nu_{*}^{\prime \prime} \rightarrow F_{q}(\tilde{X}) \rightarrow N \rightarrow 0
$$

is a split short exact sequence, and thus $\operatorname{rank} F_{q}(\tilde{X})=\operatorname{rank}\left(\operatorname{Ker} \nu_{*}^{\prime \prime}\right)$ $+\operatorname{rank} N$. Since $H_{q}(X ; \Gamma) \approx H^{n-q}(X)$, this implies $\tilde{\rho}_{q}=\rho_{q}+\rho_{n-q}$.

3. The isomorphism $H_{q}(X ; \Gamma) \approx H^{n-q}(X)$ mentioned in the proof of Theorem (4) is the local-coefficient version of the Poincare duality for orientable manifolds. Since $\tilde{X}$ is orientable, $H_{q}(\tilde{X}) \approx H^{n-q}(\tilde{X})$. In this section we point out a duality relation for the remaining group $H_{q}(X)$ in the sequence (3) and show that the exact sequence of the dual cohomology groups is the dual of the exact sequence (3).

Dual to the sequences (2) and (3) we have the (exact) sequences $0 \rightarrow \operatorname{Hom}(\Delta(X ; \Gamma), Z) \approx \Delta^{*}(X ; \operatorname{Hom}(\Gamma, Z)) \rightarrow \operatorname{Hom}(\Delta(\tilde{X}), Z)$ $\rightarrow \operatorname{Hom}(\Delta(X), Z) \rightarrow 0$ and 


$$
\begin{aligned}
\cdots & \rightarrow H^{n-q}(X ; \operatorname{Hom}(\Gamma, Z)) \stackrel{\nu^{*}}{\rightarrow} H^{n-q}(\tilde{X}) \stackrel{\mu^{*}}{\rightarrow} H^{n-q}(X) \\
& \stackrel{\delta^{*}}{\rightarrow} H^{n-q+1}(X ; \operatorname{Hom}(\Gamma, Z)) \rightarrow \cdots
\end{aligned}
$$

Recall that the isomorphism $\gamma_{\tilde{u}}: H_{q}(\tilde{X}) \approx H^{n-q}(\tilde{X})$ is given by the slant product $\gamma_{\tilde{u}}(\tilde{z})=[\tilde{U} \mid \tilde{X} \times \tilde{X}] / \tilde{z}$ where $\tilde{U}$ is an orientation of $\tilde{X}$. Likewise the isomorphism $\gamma_{u}: H_{q}(X ; \Gamma) \approx H^{n-q}(X)$ is given by slant product $\gamma_{u}(z)=[U \mid X \times X] / z$ where $U$ is the Thom class of $X$.

We may define a homomorphism $H_{q}(X) \rightarrow H^{n-q}(X ; \operatorname{Hom}(\Gamma, Z))$ as follows. First note that $H^{0}(x ; \operatorname{Hom}(\Gamma, Z)) \approx \operatorname{Hom}\left(H_{n}(X, X-x), Z\right)$. Let $\bar{U}$ be the element in $H^{n}(X \times X, X \times X-\delta X ; Z \times \operatorname{Hom}(\Gamma, Z))$ such that for each generator $z \in H_{n}(X, X-x),\langle[U \mid x \times(X, X-x)] / z, z\rangle=1$ for all $x \in X$. Now define $\gamma_{\bar{u}}: H_{q}(X) \rightarrow H^{n-q}(X ; \operatorname{Hom}(\Gamma, Z))$ by $\gamma_{\bar{u}}(z)=[\bar{U} \mid X \times X] / z$.

Thus we have the diagram

$$
\begin{aligned}
& \cdots \rightarrow H_{q+1}(\tilde{X}) \stackrel{\nu *}{\longrightarrow} H_{q+1}(X ; \Gamma) \stackrel{\partial_{*}}{\longrightarrow} H_{q}(X) \stackrel{\mu_{*}}{\longrightarrow} H_{q}(\tilde{X}) \stackrel{\nu *}{\longrightarrow} H_{q}(X ; \Gamma) \rightarrow \cdots
\end{aligned}
$$

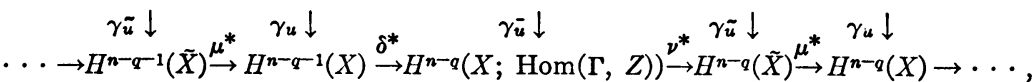

and a computation shows that the rectangles commute up to sign. By the five lemma, $\gamma_{\bar{u}}$ is bijective.

REMARKs. (i) Actually, the Thom class of $X$ uniquely determines an orientation of $\tilde{X}$; with this choice of $\gamma_{\tilde{u}}$ the diagram above is commutative.

(ii) In [1], B. Eckmann showed that, for triangulable spaces and coefficient group a field, the homology of a regular covering is determined by the homology of the base space and the group of covering transformations. In our case of the double covering $p: \tilde{X} \rightarrow X$ the relation $2 \rho_{q}=\tilde{\rho}_{q}+\operatorname{Tr} h_{q}$, where $p h=p$, may also be established for integral coefficients and without the assumption of triangulability, and implies the formula of Theorem (4).

\section{BIBLIOGRAPHY}

1. B. Eckmann, Coverings and Betti numbers, Bull. Amer. Math. Soc. 55 (1949), 95-101.

2. S. Eilenberg and N. Steenrod, Foundations of Algebraic topology, Princeton Univ. Press, Princeton, N. J., 1952.

3. H. Seifert and W. Threlfall, Lehrbuch der Topologie, Chelsea, New York, 1947.

4. E. Spanier, Algebraic topology, McGraw-Hill, New York, 1966.

5. N. Steenrod, Homology with local coefficients, Ann. of Math. 44 (1943), 610-627.

University of ArKansas 